\title{
Free-space optical communication technologies will enable next generation of ultra high throughput satellite
}

Jean-Didier Gayrard, Anaëlle Maho, Michel Sotom

Jean-Didier Gayrard, Anaëlle Maho, Michel Sotom, "Free-space optical communication technologies will enable next generation of ultra high throughput satellite," Proc. SPIE 11852, International Conference on Space Optics - ICSO 2020, 118525V (11 June 2021); doi: 10.1117/12.2600017

SPIE Event: International Conference on Space Optics - ICSO 2021, 2021, Online Only 


\section{International Conference on Space Optics-ICSO 2020}

Virtual Conference

30 March-2 April 2021

Edited by Bruno Cugny, Zoran Sodnik, and Nikos Karafolas
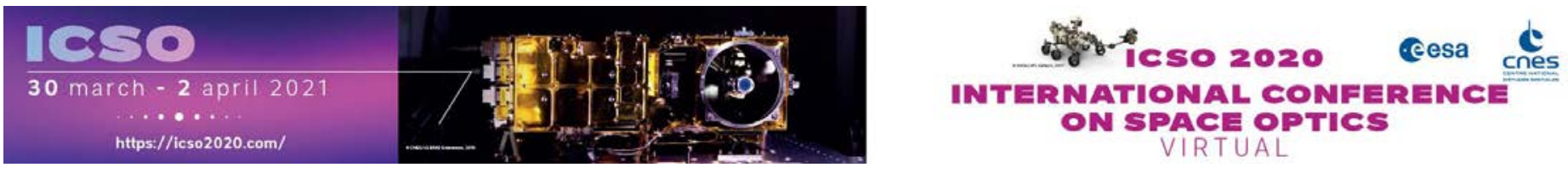

\section{Free-space optical communication technologies will enable next generation of ultra high throughput satellite}

\section{Cesa isoporecestings denes}




\title{
Free-space optical communication technologies will enable next generation of Ultra High Throughput Satellite
}

\author{
Jean-Didier Gayrard, Anaëlle Maho, Michel Sotom \\ Thales Alenia Space, 26, avenue J-F Champollion, BP 1187, 31037 Toulouse, France
}

\begin{abstract}
Ultra or Very High Throughput Satellites (U/VHTS), operating in the Ka-band (20/30GHz), are very large satellites with multi-beam coverage able to provide several hundreds of Gigabits up to few Terabits per second of aggregated throughput. The supply of these huge amounts of data to such a satellite is done by hundreds of wideband RF links, called feeder links, which operate in Ka-band $(30 \mathrm{GHz})$ and in $\mathrm{Q} / \mathrm{V}$-band $(40 / 50 \mathrm{GHz})$ between the satellite and a network of hundreds of ground (feeder/gateway) stations. Moreover, the poor availability of Q/V-band links in wet regions (such as Europe) requires additional back-up gateways (site diversity technique), further increasing the size of the ground network. In short, the cost of developing and operating such large station network becomes the major part of the cost of a VHTS and could become an obstacle to the development of the future generation of VHTS whose ambition is to exceed the Terabit of capacity. Today, none of the known techniques to reduce the number of gateway/feeder stations gives full satisfaction and have enough room for improvement. Thanks to an ESA supported study, nicknamed MATRIX for "Innovative fractionated satellite system enabling higher reuse of frequency", Thales Alenia Space studied a very promising solution. The technical and economic feasibility of this new solution based on the concept of fractionation applied here to VHTS and on free space optical communication technologies, was demonstrated. The key feature of fractionated VHTS is Optical Inter-Satellite Links (OISL) that backhaul data intended to users from the Feeder Satellites to the User Satellite. Advantageously, these OISL makes use of analogue RF modulation of optical carriers. So, it allows deporting most of signal processing in the Feeder Satellites and therefore simplifying and alleviating the User Satellite. Finally, fractionated VHTS will allow a smooth and risk-free transition from RF feeder links to digital FSO feeder links. This paper presents the concept of fractionated VHTS as developed in the MATRIX study and highlights the role and benefits of FSO communication technologies in the development and operation of future Ultra High Throughput Satellite systems. It will focus on the Optical Inter-satellite Links, cornerstone of the system.
\end{abstract}

Keywords: VHTS, FSO, OISL, feeder segment

\section{INTRODUCTION}

The economic requirements of the telecom market and the competition from terrestrial networks force satellite operators and manufacturers to significantly reduce the cost of satellite communications while delivering higher data rates to users. The way so far has consisted in considerably increasing the capacity of communication satellites through massive reuse of RF frequencies in the user coverage and implementation of numerous narrow antenna beams. These architectures can provide an aggregate capacity in excess of hundreds of Gbps reaching recently the "Terabit Satellite"[1]. The role of the so-called "feeder" segment is to provide the satellite with data intended for users. In this architecture, the supply of data to the satellite is done by a set of gateway stations or feeder stations. In transparent VHTS, the bandwidth of the feeder link, equal to the bandwidth offered by the satellite to the users, is therefore several hundred GHz wide. The too narrow frequency bands allocated to feeder links by the International Telecommunication Union (ITU), partly in Ka-band and exclusively in V-band, forces the deployment of a large number of feeder stations (several dozens). Moreover, the poor link availability due to the European climate in these very high frequencies requires the use of the technique of site diversity ( $\mathrm{N}$ nominal stations $+\mathrm{P}$ redundant stations) and thus the deployment of $\mathrm{P}$ additional stations. To the cost of these dozens of stations, it is necessary to add the cost of their interconnection by a very high speed terrestrial network (optical fiber) that we call distribution or diversity network. This feeder segment has become the bottleneck of any Very High Throughput System (VHTS) and represents a large part of the VHTS cost, exceeding that of the space segment. Faced with this state of affairs, satellite operators are seeking to reduce the Total Cost of Ownership of the feeder segment. 
There are mainly two possibilities to reduce the cost of the feeder segment: one is to reduce its outline typically by minimizing the number of sites and stations, which is essentially done by increasing the bandwidth of each feeder link; the other is discounting its cost by a progressive commissioning of the stations to closely follow the increase in satellite traffic.

\section{HOW TO INCREASE THE CAPACITY OF THE FEEDER LINKS}

Several ways are currently considered to increase the bandwidth of the feeder link and therefore to reduce the complexity and cost of the feeder segment. A first category of solution consists in increasing the bandwidth of the link.

For Radio-Frequency links, it is a question of using higher frequency bands such as the Q/V-band and beyond. The uplink is the most demanding in bandwidth. VHTS feeder link will typically operate in the V/Q frequency bands allocated by ITU to Fixed Satellite Service (FSS): $4 \mathrm{GHz}$ are available. More the next generation of VHTS will benefit from the opportunity of a $1 \mathrm{GHz}$ extension in V-band granted at the World Radio-communication Conference 2019 (WRC-19). Another solution is about scavenging pieces of unused Ka-band bandwidths. It is thus possible to increase the band of the feeder link by $1.5 \mathrm{GHz}$ but it requires operating dual-frequency $\mathrm{Ka} / \mathrm{V}$-band stations. But and finally, these two opportunities allow the number of stations to be reduced by only $38 \%$.

The other way to increase the bandwidth is to switch to free space optical link (FSO). The optical link has a very large bandwidth ( 15 Terahertz in the $1.550 \mathrm{~nm}$ infrared band) and very few optical feeder links (possibly only one) are needed at a time to feed a single VHTS. However this technique has a drawback: laser links are blocked by clouds. It is necessary to implement site diversity to guarantee acceptable link availability. The number and location of optical feeder stations are no longer constrained by considerations on frequency re-use or regulation issues but only on climatic conditions. Several studies have shown that a dozen stations in Europe (preferably in the south) can feed a VHTS in the 500-Gbps class with the required availability [2], [3]. As the satellite is accessed by a single station, all the traffic must be routed to the active station and the diversity network must be sized accordingly and consequently will be more expensive. This technique alone does not drastically reduce the cost of the feeder segment, but as we will see later, it takes part in it.

There are other techniques: for example the on-board regeneration of the feeder uplink. By regenerating the feeder uplink signals, we can optimize the throughput of the feeder link by using a highly spectral efficient waveform (b/s/Hz). A larger spectral efficiency reduces the required bandwidth of the feeder link and consequently the number of stations. This technique requires a digital on-board processor (OBP) which could prove to be expensive (cost of the equipment, increased mass and power consumption of the payload). However, note that an OBP also brings very interesting functional and operational advantages; moreover the coupling to an optical link is efficient and seamless, as we will see later.

All these solutions are very interesting but do not have enough room for improvement, and they do not offer a real breakthrough. As we will see later, the concept of fractionation associated with technologies of free space optical communications can bring in this expected breakthrough for the next generation of VHTS.

\section{THE FRACTIONNATION AND THE MATRIX PRODUCT}

The proposed fractionation concept aims at reducing the complexity of the feeder segment and to support its progressive deployment over time, thus providing an innovative and very attractive response. Satellite fractionation consists in distributing the functions usually performed by a single communication satellite among a cluster of geosynchronous satellites, namely several Feeder Satellites being interconnected to one User Satellite by means of optical inter-satellite links (OISL). This concept has been assessed as a potential solution for flexible VHTS within the ESA MATRIX contract (Innovative fractionated satellite system enabling higher reuse of frequency). Two patents are at the origin of this new concept [8], [9].

The interest for fractionated spacecraft began in the early 2000s [4], [5], [6]. A fractionated spacecraft is a network of satellites resulting from the distribution of functions performed by a single satellite among distinct co-located satellites interconnected by inter-satellite links. Here, a fractionated VHTS is therefore a cluster of GSO satellites interconnected by Inter-Satellite Links (ISL) that provides broadband communications as does a single "monolithic" VHTS. The fractionation consists of separating the feeder function (links between gateways and satellite) that will be provided by 
several Feeder Satellites, from the user function (link between satellite and user terminals) that will be done by User Satellites. So, a fractionated GEO VHTS, which we call MATRIX system or MATRIX product, is constituted of:

- A GEO User Satellite is equipped to communicate with the user terminals located inside user beams through bidirectional user links (forward/return) in Ka-band,

- A set of GSO Feeder Satellites are equipped to communicate with a set of feeder stations through bidirectional feeder links and to the USat through bidirectional OISL.
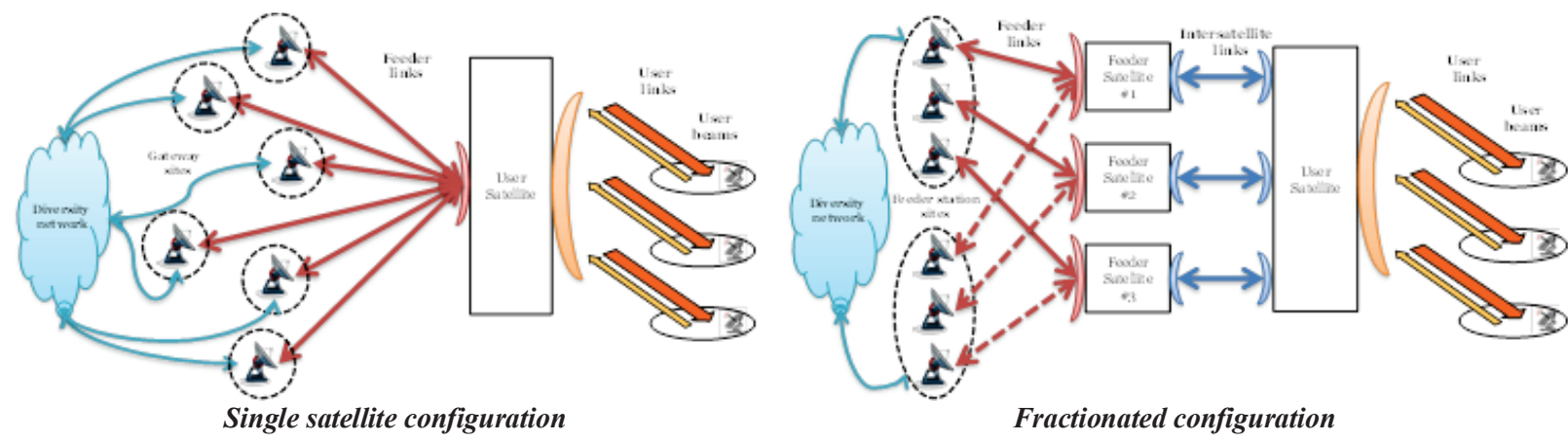

Figure 1: Principle of VHTS fractionation

The MATRIX system must be seen as a new "product". It can be presented as the next generation (later than 2025) of massive (> 500 Gbps) Satellite Broadband Internet Access systems (VHTS) operating in the Ka-band. The MATRIX product is a set of building blocks that are standard and interoperable. Any space segment can be designed using three standard building blocks:

- the USat (for User Satellite) is a standardized satellite with a throughput of 750 Gbps to the users,

- the RF-FSat (for Radiofrequency Feeder Satellite), also standardized, provides RF feeder links with 11 feeder stations,

- the O-FSat (for Optical FSat), also standardized, provides a single optical feeder link with an optical ground station.

A standardized Optical Inter-Satellite Link (OILS) air interface is the main feature of the MATRIX product. It allows any type of Feeder Satellites (RF-FSat, O-FSat) to communicate with any User Satellites (USat).

The MATRIX product has been designed to offer two types of VHT Systems: Transparent systems using RF feeder links and bent-pipe satellites and Regenerative systems using FSO feeder links and regenerative satellites.

\section{Transparent systems:}

These are the current generation of VHTS systems. The space segment is transparent. The feeder link is RF $(\mathrm{Q} / \mathrm{V}-\& \mathrm{Ka}-$ band). DVB Modem (DVB-S2X, DVB-RCS2) are in the feeder stations. Digital Transparent Processor (DTP) on board the Feeder Satellites (RF-Fsat) brings flexibility in the allocation of feeder stations to the user beams, allowing a progressive deployment of the stations.

Regenerative systems:

These systems are surely the next generation of VHTS. The space segment is fully regenerative; it includes the DVB modems on board the Feeder Satellites (O-Fsat). The feeder links are based on digital FSO or laser communications. Feeder stations are transparent and they relay Internet data to the Feeder Satellites where the data is DVB modulated on RF carriers. These modulated data are then relayed by the User satellite to the user terminals.
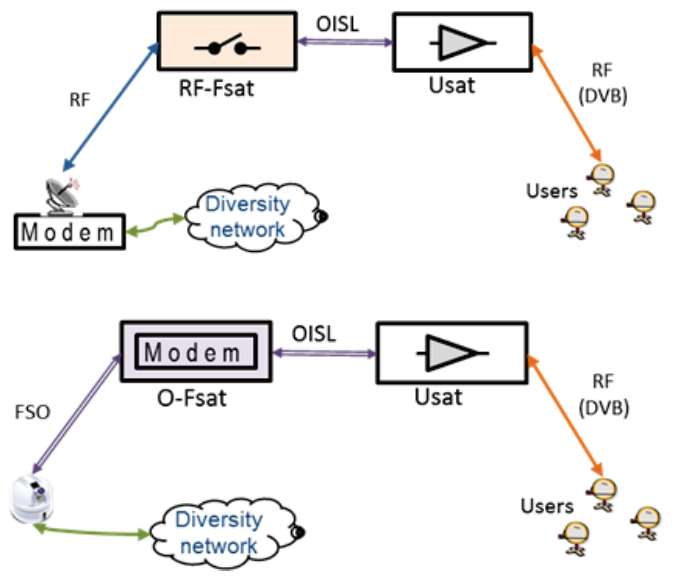

Figure 2: Two types of VHTS 


\section{THE OPTICAL INTERSATELLITE LINKS}

The optical inter-satellite links between the User Satellite and the Feeder Satellites are the main and essential feature of the MATRIX product. Theirs functions are: on the forward mission (Internet to users) to transmit from the Feeder Satellites to the User Satellite the signals from the feeder stations and for the return mission (users to Internet) to transmit from the User Satellite to the Feeder Satellites the signals from the user terminals.

A key feature of the MATRIX product is that no signal processing is performed on board the User Satellite. So all processing are performed on-board the Feeder Satellites. Either it is transparent routing between feeder stations and user beams which will therefore take place in the Feeder Satellites usually within a Digital Transparent Processor or DTP (case of the RF-FSat). Or it is regeneration of the feeder link signals and switching toward the user beams which will therefore take place in the Feeder Satellites within a Digital On-Board Processor or OBP (case of the O-FSat). As a result, the User Satellite does not have any signal processing and its functionality is limited to routing the signals from the user beams to the different Feeder Satellites and vice-versa. To allow these this particular characteristic, the idea is to transmit between the Feeder Satellites and the User Satellite the spectral content of each of the user spot beams (see fig. $3)$.

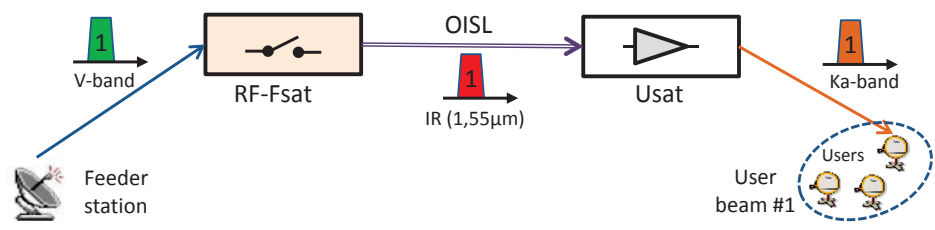

Figure 3: Principle of transmission of the beam \#1 spectral content from the feeder station to the users inside User beam \#1
An interesting sizing case is the following: the User Satellite is designed to handle 168 user spot beams without any kind of processing on board (without DTP or OBP). An user beam has a bandwidth of $1.5 \mathrm{GHz}$ in transmit (forward) and $0.5 \mathrm{GHz}$ in receive (return), which gives a total capacity for the User Satellite of $252 \mathrm{GHz}$ forward and $84 \mathrm{GHz}$ return.

To allow a gradual deployment of Feeder Satellites, OISLs are designed to support the full capacity, i.e. $252 \mathrm{GHz}$ (168 spots x $1.5 \mathrm{GHz})$ on forward direction, and $84 \mathrm{GHz}(168$ spots x $0.5 \mathrm{GHz})$ in return direction. These bandwidths $(252$ $\mathrm{GHz}$ and $84 \mathrm{GHz}$ ) are transmitted by OISL using analogue RF modulation of optical carriers. The forward bandwidth $(252 \mathrm{GHz})$ is cut into 42 sub-bands of $6 \mathrm{GHz}$ corresponding to the spectral content of 4 user beams. Each of these 42 $6 \mathrm{GHz}$-bands is analogically modulating a wavelength (optical carrier). Likewise, the return bandwidth (84 GHz) is cut into 28 sub-bands of $3 \mathrm{GHz}$ corresponding to the spectral content of 6 user beams. Each of these $283 \mathrm{GHz}$-bands is analogically modulating a wavelength. Figures $3 \& 4$ below show examples of the RF spectrum of the optical channels received and transmitted at the User Satellite.

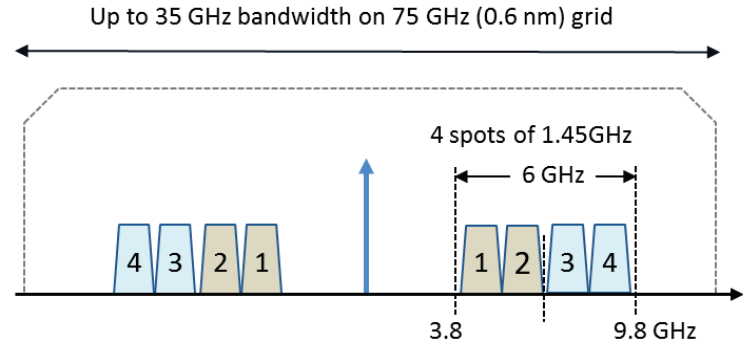

Figure 4: Spectral content of forward OISL channels (wavelength or optical carrier) received at USat

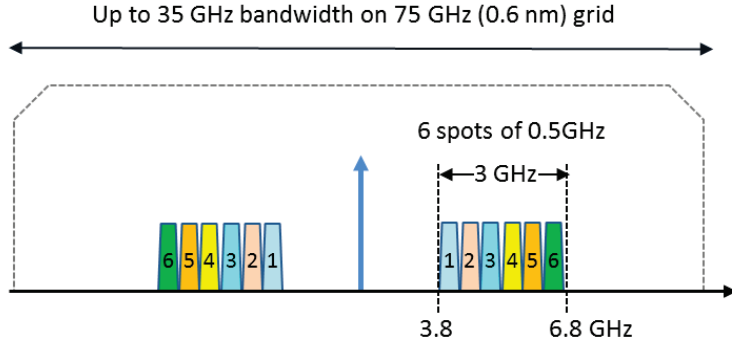

Figure 5: Spectral content of return OISL channels (wavelength) transmitted at USat

So the User Satellite is equipped to handle three OISL with the three Feeder Satellites, and each Feeder Satellite is equipped to operate a single OISL. The User Satellite payload includes one optical terminal equipped with 3 optical heads pointing at feeder satellites, so as to support 3 independent bi-directional OISL, one from/to each of the 3 Feeder Satellites. The optical head units are equipped with a telescope of $15 \mathrm{~cm}$ diameter aperture. The Feeder Satellite payload includes one optical terminal pointing at the User Satellite, and supporting one bi-directional OISL from/to the User Satellite. This optical head unit is also equipped with a telescope of $15 \mathrm{~cm}$ diameter aperture. To ensure gradual deployment, Feeder Satellites are designed to handle the full bandwidth or spectrum of the mission $(252 \mathrm{GHz}+84 \mathrm{GHz})$. 
However, each Feeder Satellite only handles a maximum of a third of the traffic, so the power sizing of these satellites and their OISLs is made on this assumption of a third of traffic.

The figure 6 shows a possible wavelength allocation plan of the OISL. The optical carriers are implemented by using the HDWDM technique with a channel spacing of $75 \mathrm{GHz}$ (or $0.6 \mathrm{~nm}$ ). Forward and return channels or carriers are implemented respectively in C-band and L-band and separated by a guard band of about $10 \mathrm{~nm}$.

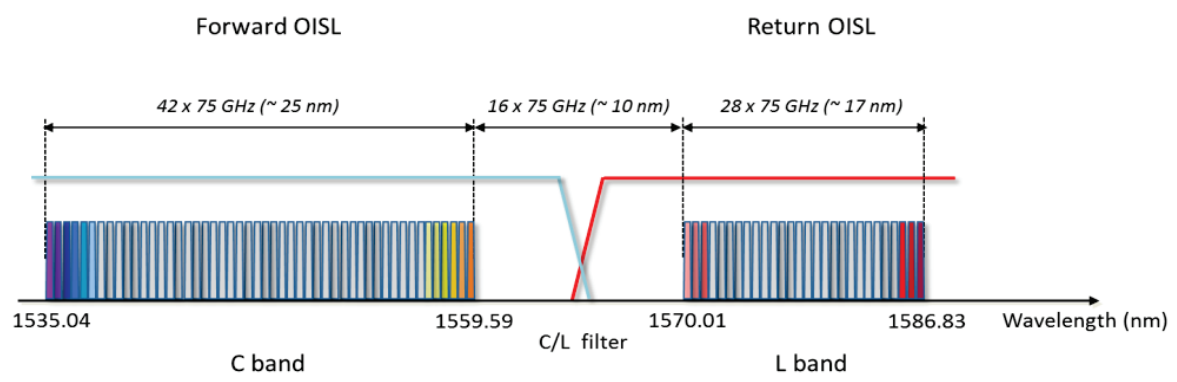

Figure 6: Example of wavelength allocation plan of forward \& return Optical ISL

The power dimensioning of OISLs is constrained by several elements. The first element is the requirement in Signal-toNoise and Intermodulation Ratio (SNIR) to be realized on the optical link in order to obtain an end-to-end SNIR which shall be compatible with the Internet access mission at the user terminal. The second element is the link power budget on the OISL that takes into account the optical terminals gains $(\varnothing 15 \mathrm{~cm}$ aperture) and the distance between the satellites (typ. $1500 \mathrm{~km}$ ). Another element is the power handling capacity of the transmission hardware (Wavelength Division Multiplexer (WDM), High Power Optical Amplifiers (HPOA)...).

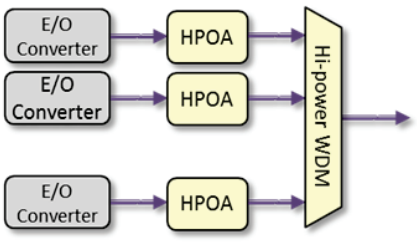

Architecture A

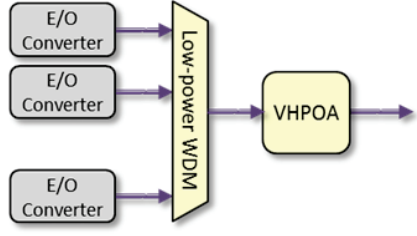

Architecture B

Multiplexing 42 wavelength or carriers onto a single link is also a critical aspect of the design of the feeder satellite. Two example of optical architectures are considered for multiplexing DWDM channels and delivering the required power to each channel for the forward OISL (see fig 5.). Architecture \#A makes use of multiple HPOA in parallel and a high-power WDM at the output. Instead Architecture \#B makes use of a low-power WDM and a veryhigh-power optical amplifier (VHPOA) at the output.

Both Feeder Satellites and User Satellite are equipped with the same optical inter-satellite link terminal which includes optical heads (telescope) with a diameter of $15 \mathrm{~cm}$. This unit could be derived from the OISL terminal that was developed by Thales Alenia Space - Switzerland for LEO constellation and called OPTEL-C. This equipment is the heir to Thales Alenia Space's long experience in the development of optical terminals [10].

The OISL are instrumental to the MATRIX system. Thanks to analog modulation of optical carriers by the spectral contents of user beams, no signal processing is required on board the User Satellite. The User Satellite is thus optimized to offer the maximum capacity (bandwidth) on the Ka-band direct link to users. This also makes it possible to

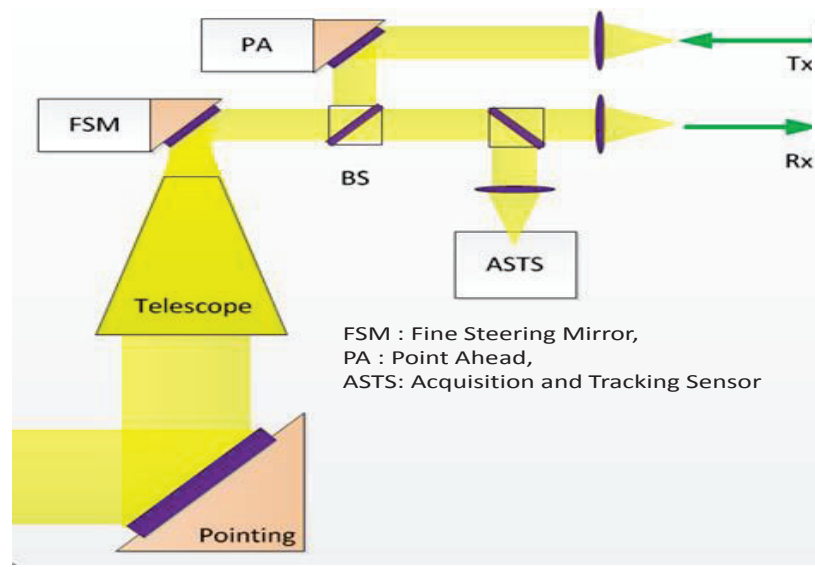

Figure 8: Wavelength multiplexing architectures standardize the User Satellite and to reduce production costs. Finally, being transparent, the OISL allows deporting all processing in the Feeder Satellites. It is then possible to mix the two types of Feeder Satellites (RF-FSat and O-FSat) and therefore to allow an upgrade from a transparent radio system to a regenerative optical system. 


\section{THE OPTICAL FEEDER LINK}

The second utilization of free space optical links in the MATRIX product is the optical feeder links of the regenerative VHTS systems (see fig. 2). The optical feeder links between ground optical feeder stations and Feeder Satellites are used to directly relay Internet data to DVB modulators on board the Feeder Satellites. These data once modulated in DVB are relayed transparently by the User Satellite toward the user terminals. It is clear that in future generations of regenerative VHTS, free space optical link technologies will be paramount. As a key feature of the MATRIX product is that no signal processing is performed in User Satellites, it means that data demodulation / modulation and switching are performed onboard the Feeder Satellites within a Digital On-Board Processor (O-FSat). The optical feeder link uses a digital modulation of the optical carriers.

In the interesting sizing case where the User Satellite handles 168 user spot beams (1.5GHz forward and $0.5 \mathrm{GHz}$ return), the maximum total throughput is $540 \mathrm{Gbps}$ forward and $120 \mathrm{Gbps}$ return, totalling a capacity of 660 Gbps. Note, this corresponds to a spectral efficiency of $2.4 \mathrm{~b} / \mathrm{s} / \mathrm{Hz}$ on downlink and $1.8 \mathrm{~b} / \mathrm{s} / \mathrm{Hz}$ on uplink.

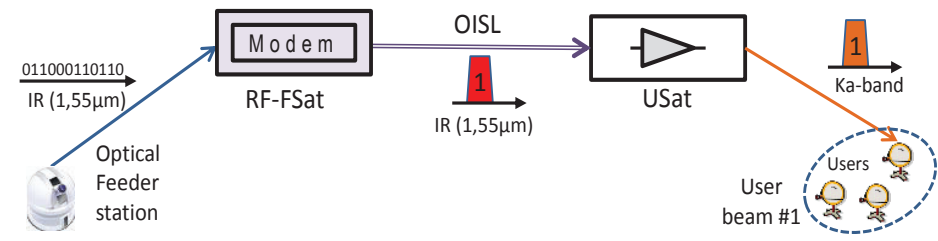

Figure 9. Principle to transmit data from the optical feeder station to users inside User beam \#1
In an Optical Feeder Satellite (O-FSat), the digital optical feeder link supports the feeder function of one-third of the full capacity, i.e. 180 Gbps uplink (forward) on 18 wavelengths or carriers $(\lambda)$ modulated at 10 -Gbps and 40 Gbps downlink (return) as 4 wavelengths of 10 Gbps. The technique of digital modulation of optical carriers (wavelength) is for example the Differential Phase Shift Keying (DPSK) format as we will see later.

Figure 10 shows a possible wavelength allocation of the optical feeder link. Wavelength are implemented according to the DWDM technique with a $100 \mathrm{GHz}$ $(0.8 \mathrm{~nm})$ channel spacing. Up and downlink channels are respectively implemented in blue and red subbands of C-band and separated by a $10 \mathrm{~nm}$ guard band. In addition, orthogonal polarizations (RHCP \& LHCP) could be used to enhance uplink/ downlink channel isolation.

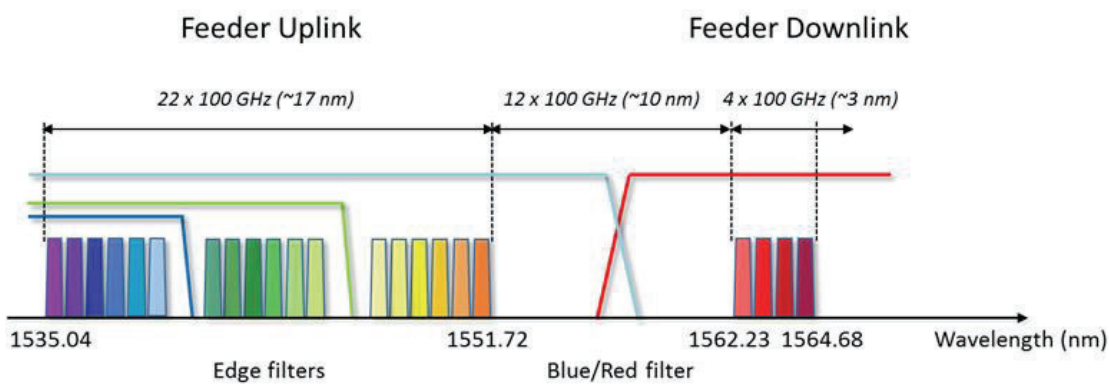

Figure 10: Example of wavelength allocation plan of the Optical feeder link (uplink \& downlink)

The O-FSat payload includes one optical terminal pointing at the Earth, and supporting one bi-directional optical feeder link from/to an optical ground station (OGS). The optical head unit is equipped with a telescope of $25 \mathrm{~cm}$ diameter aperture. The OGS is equipped with a telescope of $70 \mathrm{~cm}$ in diameter. The distance between the satellite and the station reaches $38310 \mathrm{~km}$.

Optical links through the Earth's atmosphere are disturbed by phenomena such as atmospheric absorption (gas), ice clouds, scintillation caused by atmospheric turbulences, scattering by atmospheric gases and aerosols, which causes losses. So provisions for atmospheric losses are taken into account in the link budget. More, water clouds, made up of water droplets, are totally blocking the light at the telecom IR wavelengths. So, in the case of the presence of water clouds (cumulus and stratus) on the path, it is not possible to establish any optical link. Site diversity methods must be implemented to ensure good availability of links ([2][3]). Despite these operating constraints, the optical feeder link is a real technical advance which will allow future generations of VHTS to be directly feed with Internet data. Optical feeder link takes all its interest in the case of the MATRIX product where the modulation on the DVB radio carriers is made on board the Feeder Satellites without taking power resources from the User Satellite. 
Figure 11 gives the theoretical BER at $10 \mathrm{Gbps}$ vs. the received optical power for three modulation and detection schemes: OOK (On-Off Keying) with Direct-Detection, DPSK (Differential Phase Shift Keying) with Differential Direct Detection (DDD) and BPSK (Binary Phase Shift Keying) with Coherent Detection.

DPSK/DDD is an attractive option as it provides a fair trade-off between required power and implementation complexity. The experimental BER curve (figure 12) shows that a sensitivity of 20 photons per bit at a

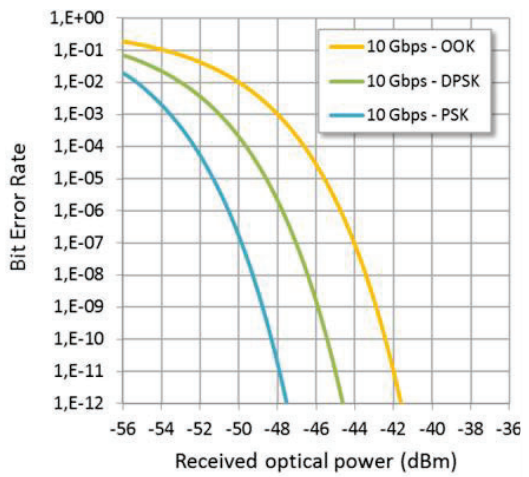

Figure 11: BER vs. Rx power

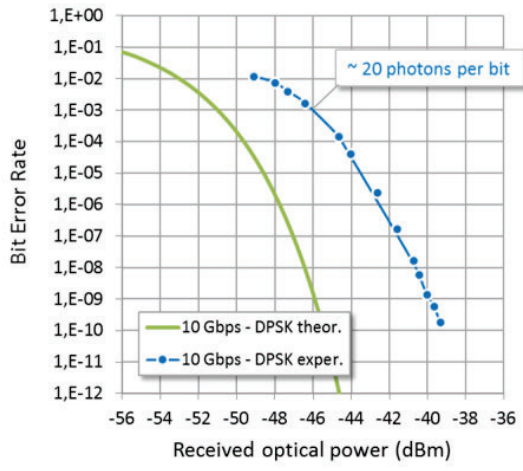

Figure 12: BER vs. Rx power (DPSK- DDD) BER of $10^{-3}$ can be achieved with DPSK.

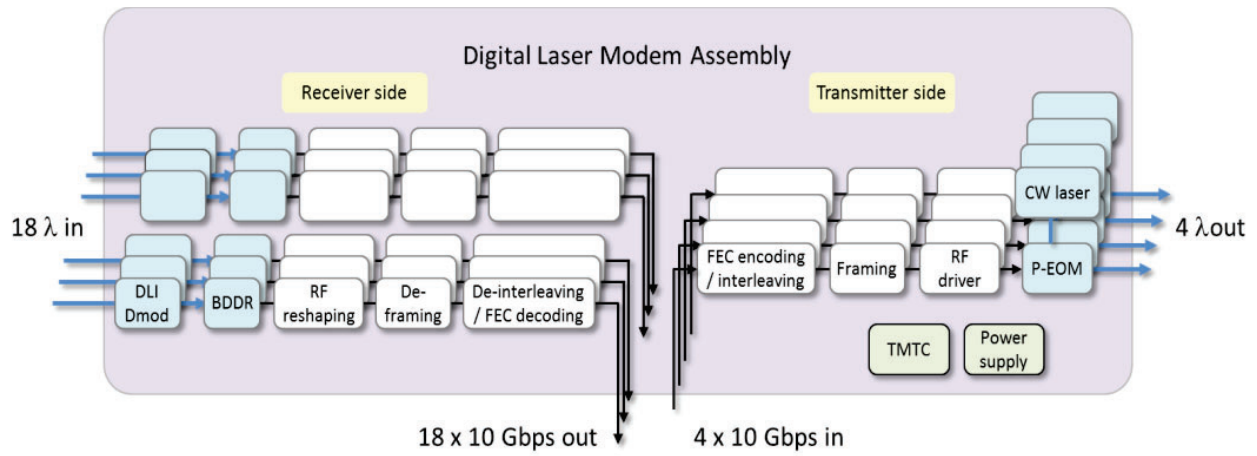

Figure 13: Block diagram of the Digital Laser Modem Assembly for Optical Feeder Link

The payload of the O-FSat includes a new type of equipment: the Digital Laser Modem Assembly. This unit interfaces between the optical terminal and the optical amplifiers on one side and the DVB-OBP on the other side. The DVB-OBP is in charge of modulating the Internet data on DVB-S2 RF carriers, which are in turn transferred (intensity modulation) onto the optical carriers of the OISL and destined for the User Satellite.

This last utilization of free space optical links in the MATRIX product is very interesting. Indeed it gives to the MATRIX system the possibility of gradually deploying (by third of the total capacity in our sizing example) a regenerative VHTS system. This minimizes for the satellite operator the risks associated with the commissioning of a new technology.

\section{CONCLUSION}

The MATRIX fractionating concept brings a new VHTS product to life for the next decade. Not only does this new product reduce the cost and complexity of the feeder segment, but also it brings new possibilities such as the progressive deployment of the feeder segment to follow the increase in traffic and such as the seamless and risk-free upgrade from a transparent VHTS fed by numerous radio feeder links to an regenerative VHTS fed by one or a few optical feeder links. To endow the MATRIX product with its extraordinary features, it will be essential to use the technology of free space optical communication links.

Indeed this technology is required to provide the system with optical inter-satellites links that are able to convey without processing all the radiofrequency spectra (the Ka-band spectral content of each user beams) between the Feeder Satellites and the User Satellite. This technology will also be necessary to commission future regenerative VHTS fed by one or a few optical feeder links. For these systems, it is a question of directly connecting the VHTS satellites to the terrestrial optical fibre networks. And this will be done thanks to the free space optical links at very high speed (hundreds of Gbps). 
DISCLAIMER: The activity presented above was carried out under the "Innovative fractionated satellite system enabling higher reuse of frequency MATRIX (Multiple Access Telecom Reconfigurable Inter-satellites X)" study, funded by the European Space Agency (ESA Contract 4000124660/18/NL/GLC). The views expressed in this publication can in no way be taken to reflect the official opinion of the European Space Agency.

\section{REFERENCES}

[1] J.-D. Gayrard, “Terabit Satellite: Myth or Reality?”, SPACOMM09, Colmar, France, 20-24 July 2009.

[2] N. Girault, P.-D. Arapoglou, "Optical Feeder Link Architectures for Very High Throughput Satellites: Ground Segment", International Conference on Space Optics, ICSO 2018, Chania, Greece, 9-12 Oct 2018.

[3] A. Mody, E. Gonzalez, "An Operator's View: The Medium-Term Feasibility of an Optical Feeder Link for VHTS" IEEE Int. Conf. on Space Optical Systems and Applications, Okinawa, Japan, 14-16 November 2017.

[4] J.-D. Gayrard, A. Cotellessa, E. Bertenyi, B. Perrot, "SkyLAN: a new approach for scalable multimedia system", 55th International Astronautical Congress, IAF, Vancouver, Canada, 4 - 8 October 2004.

[5] J.-D Gayrard, B. Perrot, E. Bertenyi, A. Cotellessa, "Are satellite clusters a good deal for commissioning broadband satellite systems?", 23rd AIAA ICSSC, Rome, Italy 25-28 September 2005.

[6] J.-D. Gayrard, G. Baister, M. Molina Cobos, A. Cotellessa, "Optical Inter-Satellite Links for SkyLAN clusters of broadband communications satellites", 23rd AIAA ICSSC, Rome, Italy, 25-28 September 2005.

[7] J.-D. Gayrard \& al., "Fractionation, a new solution for flexible Very High Throughput Satellites", 3rd ESA Workshop on Advanced Flexible Telecom Payloads, Noordwijk, The Netherlands, 21-24 March 2016

[8] J.-D. Gayrard, European patent EP 2723001B1 "High capacity satellite telecommunication system with multibeam coverage and frequency reusing", delivered on 24-Jun-2015.

[9] Jerome Tronc, Nicolas Girault, Ana Bolea Alamanac, Xavier Geneste, Michael Hadjitheodosiou, patent WO 2019/137625 A1 "Satellite communication system and method of communicating in a satellite communication system", filled by 15-Jan-2018.

[10] https://gandh.com/wp-content/uploads/2019/03/6 Buchheim TASCH OFC19-1.pdf 